\title{
The Systolic Blood Pressure Difference Between Arms and Cardiovascular Disease in the Framingham Heart Study
}

\author{
Ido Weinberg, ${ }^{a}$ Philimon Gona, ${ }^{b, c}$ Christopher J. 0'Donnell, ${ }^{\mathrm{a}, \mathrm{b}, \mathrm{d}}$ Michael R. Jaff, ${ }^{\mathrm{a}, 1}$ Joanne M. Murabito ${ }^{\mathrm{b}, \mathrm{e}, 1}$ \\ ${ }^{a}$ Institute for Heart Vascular and Stroke Care, Massachusetts General Hospital, Boston; ${ }^{b}$ National Heart, Lung and Blood Institute (NHLBI) \\ Framingham Heart Study, Framingham, Mass; ${ }^{c}$ Division of Biostatistics and Health Services Research, Department of Quantitative Health \\ Sciences, University of Massachusetts Medical School, Worcester; ${ }^{d}$ NHLBI Division of Intramural Research, Bethesda, Md; ${ }^{e}$ Section of \\ General Internal Medicine, Department of Medicine, Boston University School of Medicine, Boston, Mass.
}

\begin{abstract}
BACKGROUND: An increased interarm systolic blood pressure difference is an easily determined physical examination finding. The relationship between interarm systolic blood pressure difference and risk of future cardiovascular disease is uncertain. We described the prevalence and risk factor correlates of interarm systolic blood pressure difference in the Framingham Heart Study (FHS) original and offspring cohorts and examined the association between interarm systolic blood pressure difference and incident cardiovascular disease and all-cause mortality.

METHODS: An increased interarm systolic blood pressure difference was defined as $\geq 10 \mathrm{~mm} \mathrm{Hg}$ using the average of initial and repeat blood pressure measurements obtained in both arms. Participants were followed through 2010 for incident cardiovascular disease events. Multivariable Cox proportional hazards regression analyses were performed to investigate the effect of interarm systolic blood pressure difference on incident cardiovascular disease.

RESULTS: We examined 3390 (56.3\% female) participants aged 40 years and older, free of cardiovascular disease at baseline, mean age of 61.1 years, who attended a FHS examination between 1991 and 1994 (original cohort) and from 1995 to 1998 (offspring cohort). The mean absolute interarm systolic blood pressure difference was $4.6 \mathrm{~mm} \mathrm{Hg}$ (range 0-78). Increased interarm systolic blood pressure difference was present in $317(9.4 \%)$ participants. The median follow-up time was 13.3 years, during which time 598 participants $(17.6 \%)$ experienced a first cardiovascular event, including 83 (26.2\%) participants with interarm systolic blood pressure difference $\geq 10 \mathrm{~mm} \mathrm{Hg}$. Compared with those with normal interarm systolic blood pressure difference, participants with an elevated interarm systolic blood pressure difference were older (63.0 years vs 60.9 years), had a greater prevalence of diabetes mellitus (13.3\% vs 7.5\%,), higher systolic blood pressure (136.3 mm Hg vs $129.3 \mathrm{~mm} \mathrm{Hg})$, and a higher total cholesterol level $(212.1 \mathrm{mg} / \mathrm{dL}$ vs $206.5 \mathrm{mg} / \mathrm{dL}$ ). Interarm systolic blood pressure difference was associated with a significantly increased hazard of incident cardiovascular events in the multivariable adjusted model (hazard ratio 1.38; 95\% CI, 1.09-1.75). For each 1-SD-unit increase in absolute interarm systolic blood pressure difference, the hazard ratio for incident cardiovascular events was 1.07 (95\% CI, 1.00-1.14) in the fully adjusted model. There was no such association with mortality (hazard ratio 1.02 ; $95 \%$ CI $0.76-1.38$ ).

CONCLUSIONS: In this community-based cohort, an interarm systolic blood pressure difference is common and associated with a significant increased risk for future cardiovascular events, even when the absolute difference in arm systolic blood pressure is modest. These findings support research to expand clinical use of this simple measurement.
\end{abstract}

(C) 2014 Elsevier Inc. All rights reserved. • The American Journal of Medicine (2014) 127, 209-215

KEYWORDS: Cardiovascular disease; Cardiovascular risk; Interarm blood pressure difference

Funding: See last page of article.

Conflicts of Interest: JMM is a board member of Vascular Interventional Advances (VIVA) Physicians, a 501c3 nonprofit education and research organization; all other authors have no potential conflicts of interest to disclose.

Authorship: See last page of article.
Requests for reprints should be addressed to Michael R. Jaff, DO, Institute for Heart Vascular and Stroke Care, Massachusetts General Hospital, 55 Fruit St., Warren 905, Boston, MA 02114.

E-mail address: mjaff@partners.org

${ }^{1}$ These authors contributed equally to the manuscript. 
An increased interarm systolic blood pressure difference is usually defined as $10 \mathrm{~mm} \mathrm{Hg}$ or greater ${ }^{1,2}$ and can be found in up to $24 \%$ of healthy individuals. ${ }^{3-6}$ It has been suggested that identification of interarm systolic blood pressure difference is crucial for appropriate identification and treatment of hypertension, ${ }^{7-9}$ for clinical decision-making and surveillance. $^{1,10-13}$ Interarm systolic blood pressure differences have been studied in patients in primary care settings, ${ }^{14-16}$ in patients with various manifestations of vascular disease, ${ }^{17-19}$ and in populationbased cohorts. $^{3,6,16}$ It has been found to correlate with classic and novel cardiovascular risk factors and may be predictive of cardiovascular events. Nevertheless, data on the epidemiology of interarm systolic blood pressure difference are relatively scarce. ${ }^{3,6,16,17}$ While

\section{CLINICAL SIGNIFICANCE}

- Interarm systolic blood pressure difference (ISBPD) is associated with incident cardiovascular events independent of traditional cardiovascular risk factors in a large community-based cohort.

- Measurement of blood pressure in both arms is important both for accurate blood pressure detection and for cardiovascular risk stratification.

\section{an association between interarm}

systolic blood pressure difference and mortality was noted in 3 small prospective studies and a meta-analysis that pooled data from 20 older studies, ${ }^{14,15,17,18,20}$ the evidence for an association with cardiovascular disease is less consistent. ${ }^{20}$ The objectives of this study were to describe the distribution of interarm systolic blood pressure difference and risk factor correlates in the Framingham Heart Study (FHS) original and offspring cohorts and to determine the association between interarm systolic blood pressure difference and incident cardiovascular events and all-cause mortality.

\section{METHODS}

\section{Study Participant Sample}

Methods of recruitment have been described previously for participants from the original cohort and the offspring study cohort of the FHS. ${ }^{21,22}$

From the original FHS cohort of 5209 that was recruited in $1948,{ }^{23} 1026$ men and women attended an FHS examination between 1991 and 1994. There also were 3532 men and women from the offspring study cohort who attended the sixth offspring cycle examination from 1995 to 1998. Of the 4558 total original and offspring cohort participants, 494 were excluded because they did not have a blood pressure measurement in both arms. Of the remaining participants, 66 participants $<40$ years old and 608 with prevalent cardiovascular disease were excluded. The Institutional Review Board at Boston University Medical Center approved the examination content, and informed consent was obtained from study participants at the time of their examination.

\section{Interarm Systolic Blood Pressure Measurement}

For the purpose of calculating the interarm systolic blood pressure difference, brachial systolic blood pressure measurements were obtained as part of an ankle-brachial index

\section{Definition of Incident Cardiovascular Disease Events and Mortality}

Participants were followed through 2010 for the first incident cardiovascular event, defined as coronary heart disease (ie, a fatal coronary event, recognized myocardial infarction and unrecognized myocardial infarction, coronary insufficiency, or angina), a cerebrovascular event (ie, ischemic stroke or transient ischemic attack), intermittent claudication, ${ }^{24}$ or congestive heart failure. ${ }^{25}$ An unrecognized myocardial infarction was adjudicated when serial changes on the electrocardiogram showed development of pathologic $\mathrm{Q}$ waves and neither the participant nor the physician considered the possibility of a myocardial infarction. Mortality data were obtained by review of all medical records including the last hospitalization, nursing home records, personal physical records, and if needed, next-of kin interviews along with death certificate evaluation. All cardiovascular events and deaths in both the original cohort and offspring participants were adjudicated by an end-point committee of 3 senior investigators using the same criteria and all available medical records. Ischemic stroke and transient ischemic attack events were reviewed by a panel of study neurologists.

\section{Definition of Risk Factor Covariates}

Covariates were obtained at the same time as the interarm systolic blood pressure difference measurement. Hypertension was defined as a blood pressure $\geq 140 / 90 \mathrm{~mm} \mathrm{Hg}$ or the use of antihypertension medications. Body mass index was calculated as the participants' weight in kilograms divided by the participants' height in meters squared. Diabetes mellitus was considered present if the fasting glucose was $\geq 126 \mathrm{mg} / 100 \mathrm{~mL}$ (offspring), a nonfasting glucose of $\geq 200$ $\mathrm{mg} / \mathrm{dL}$ (original cohort), or if the participant was receiving 
treatment with insulin or oral hypoglycemic medications. Total cholesterol was analyzed as a continuous variable. Lipid-lowering therapy was entered into the model as a separate binary variable. Participants were queried at each examination about cigarette smoking, and current smoking was defined as smoking at least one cigarette per day in the year preceding the examination. The 10-year Framingham risk score for general cardiovascular disease was calculated for each participant. ${ }^{26}$

\section{Statistical Analysis}

Descriptive statistics were generated. Continuous measures were summarized using mean and SD. Categorical traits were summarized using percentages. We standardized interarm systolic blood pressure difference (ISBPD) to mean $=0$ and $\mathrm{SD}=1$ because this measure is skewed to the right. Hazard ratios (HRs) for continuous ISBPD were presented for every 1-SD-unit increase in blood pressure. The main exposure, ISBPD, was first analyzed as a binary variable (ie, $<10 \mathrm{~mm} \mathrm{Hg}$ and $\geq 10 \mathrm{~mm} \mathrm{Hg}$ ); then as a 3-level ordinal variable ( $<10 \mathrm{~mm} \mathrm{Hg}$ vs 10 to $<15 \mathrm{~mm} \mathrm{Hg}$ and $\geq 15 \mathrm{~mm} \mathrm{Hg}$ ), and then as a continuous trait. We tested for linear trend in the hazards across the 3-level ordinal variable. Two-sample $t$-test with pooled variance or unequal variance as indicated was used to compare means between participants who had ISBPD $\geq 10 \mathrm{~mm} \mathrm{Hg}$ versus those with ISBPD $<10 \mathrm{~mm} \mathrm{Hg}$. The chi-squared test was used to compare categorical traits. Age- and sex-adjusted time to first cardiovascular event was analyzed using Kaplan-Meier plots and evaluated using the log-rank chi-squared test.

Next, after confirming the validity of the Cox proportional hazards regression assumptions, 2 Cox models were fitted with ISBPD $<10 \mathrm{~mm} \mathrm{Hg}$ as the referent category. The first model adjusted for the following variables: age, sex, hypertension, total cholesterol level, high-density lipoprotein cholesterol, cholesterol treatment, presence or absence of diabetes mellitus, and smoking status; and the second model adjusted for the 10-year Framingham risk score for general cardiovascular disease. ${ }^{26} \mathrm{We}$ also assessed for linear trend using the 3-category ordinal ISBPD variable (ie, ISBPD $<10$ $\mathrm{mm} \mathrm{Hg}, 10$ to $<15 \mathrm{~mm} \mathrm{Hg}$, and $\geq 15 \mathrm{~mm} \mathrm{Hg}$ ). Furthermore, similar models were fitted, accounting for familial correlations between the original and offspring cohorts. We used generalized estimating equations to account for the familial correlations within our dataset. Similar analysis approaches were used restricting the sample to hypertensive participants and also using mortality as the outcome.

The predictive utility of the Cox model was examined by comparing the c-statistic. The incremental effect of adding ISBPD to the Framingham risk score for predicting cardiovascular outcomes was evaluated with the use of categorybased net reclassification improvement index (NRI). ${ }^{27}$ The NRI is used to assess how well an exposure "reclassifies" patients from one risk category to another. We calculated NRI using an extension to survival analysis that employs Kaplan-Meier estimates of event probabilities using categories: low risk ( 0 to $<6 \%$ ), intermediate risk ( $6 \%$ to $20 \%$ ), or high risk $(>20 \%)$. A large NRI indicates that the additional exposure causes a large improvement in reclassification.

\section{RESULTS}

Our final study sample included 3390 participants; mean age of $61.1 \pm 11.3$ years, $56.3 \%$ women, followed for an average of 13.3 years. The median ISBPD was 3 (interquartile range 2.6) $\mathrm{mm} \mathrm{Hg}$, and 317 (9.4\%) participants had an ISBPD $\geq 10 \mathrm{~mm} \mathrm{Hg}$ (Table 1). The distribution of

Table 1 Age and Sex-Adjusted Comparison of Baseline Variables by Interarm Systolic Blood Pressure Difference

\begin{tabular}{|c|c|c|c|}
\hline Variable & ISBPD $<10 \mathrm{~mm} \mathrm{Hg}\left(\mathrm{n}=3073^{*}\right)$ & ISBPD $\geq 10 \mathrm{~mm} \mathrm{Hg}\left(\mathrm{n}=317^{\star}\right)$ & $P$-Value \\
\hline Age $†$ & $60.9(11.3)$ & $63.0(11.5)$ & .002 \\
\hline Male $(\%) \dagger$ & $1343(43.7)$ & $137(43.2)$ & .87 \\
\hline Body mass index $\mathrm{kg} / \mathrm{m}^{2}$ & $27.4(4.8)$ & $29.6(6.2)$ & $<.001$ \\
\hline SBP mm Hg & $129.3(19.3)$ & $136.3(20.3)$ & $<.001$ \\
\hline DBP mm Hg & $74.9(9.8)$ & $76.3(9.9)$ & .02 \\
\hline Hypertension (\%) & $1249(40.6)$ & $184(58.0)$ & $<.001$ \\
\hline HTN treatment $(\%)$ & 789 (25.7) & $137(43.6)$ & $<.001$ \\
\hline Total cholesterol mg/dL & $206.5(39.3)$ & $212.1(40.2)$ & .17 \\
\hline HDL cholesterol mg/dL & $51.8(16.2)$ & $51.2(16.9)$ & .53 \\
\hline Triglycerides mg/dL & $137.8(135.2)$ & $151.2(105.8)$ & .004 \\
\hline Lipid lowering treatment (\%) & $282(9.2)$ & $38(12.0)$ & .10 \\
\hline Diabetes mellitus (\%) & $229(7.5)$ & $42(13.3)$ & .003 \\
\hline Current cigarette use (\%) & $426(13.9)$ & $39(12.3)$ & .44 \\
\hline Ever cigarette use $(\%)$ & $1384(45.0)$ & $137(43.2)$ & .54 \\
\hline Framingham 10 -year risk score & $12.3(10.6)$ & $15.2(12.1)$ & $<.001$ \\
\hline
\end{tabular}


systolic blood pressure difference between arms in the sample is shown in Figure 1. There were 3073, 246, and 71 participants with ISBPD of $<10 \mathrm{~mm} \mathrm{Hg}, 10$ to $<15$ $\mathrm{mm} \mathrm{Hg}$ and $\geq 15 \mathrm{~mm} \mathrm{Hg}$, respectively.

Overall, 598 participants (17.6\%) experienced a first cardiovascular event, including $83(26.2 \%)$ participants with an increased ISBPD. The overall incidence rate of total cardiovascular disease was 17.64 per 1000 person-years (95\% confidence interval [CI], 16.25-19.11). Age- and sex-adjusted Kaplan-Meier plots stratified by binary ISBPD $(<10 \mathrm{~mm} \mathrm{Hg}$ vs $\geq 10 \mathrm{~mm} \mathrm{Hg}$ ) are shown in Figure 2 and show that the ISBPD $\geq 10 \mathrm{~mm} \mathrm{Hg}$ category had a significantly higher probability for incident cardiovascular event than the ISBPD $<10 \mathrm{~mm} \mathrm{Hg}$ category (log-rank test chi-squared $=18.2, P<.0001$ ).

An ISBPD $\geq 10 \mathrm{~mm} \mathrm{Hg}$ was associated with a significantly increased hazard of incident cardiovascular events in the multivariable adjusted Cox models (HR 1.38; $95 \%$ CI, 1.09-1.75) (Table 2). There was no change in the hazard with adjustment for Framingham risk score. In a multivariable model, an ISBPD $\geq 15 \mathrm{~mm} \mathrm{Hg}$ was associated with an increased hazard of incident cardiovascular events (HR 1.47; 95\% CI, 0.93-2.32). There was a statistically significant linear trend with increasing ISBPD category $(P=.009$ in the multivariable adjusted model). An ISBPD $\geq 10 \mathrm{~mm} \mathrm{Hg}$ also was associated with a significantly increased hazard of incident cardiovascular events in a subgroup analysis of 1433 hypertensive participants (HR $1.50 ; 95 \% \mathrm{CI}, 1.14-1.97$, in the multivariable model). In similar analyses for total mortality, there were no significant associations (Table 2). For each 1-SD increase in absolute ISBPD, the HR for incident cardiovascular events was 1.07 (95\% CI, 1.00-1.14) in the multivariable-adjusted model

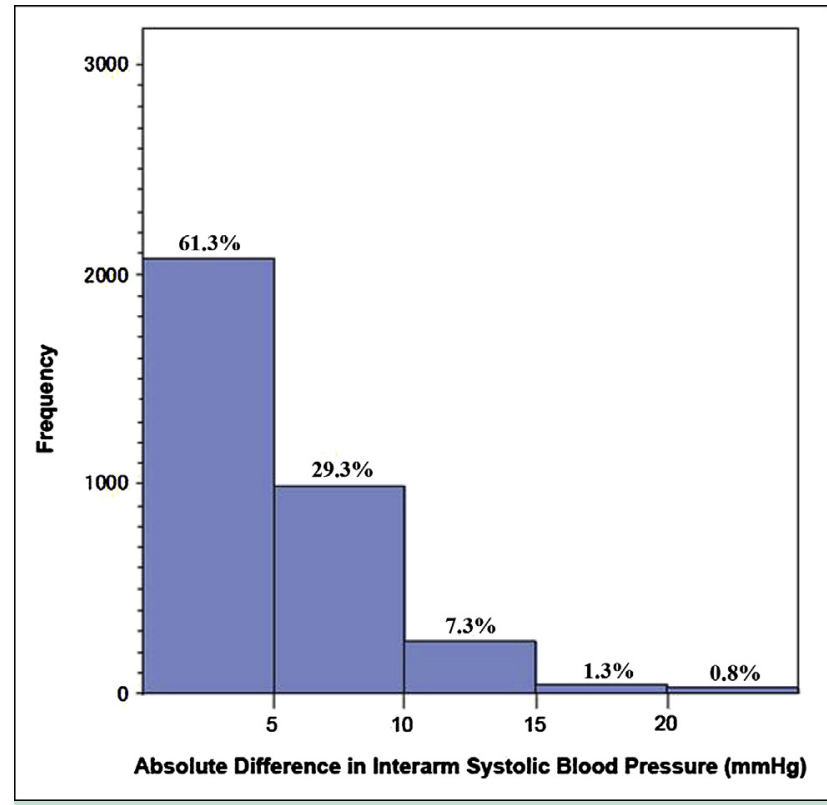

Figure 1 Distribution of absolute interarm systolic blood pressure difference.

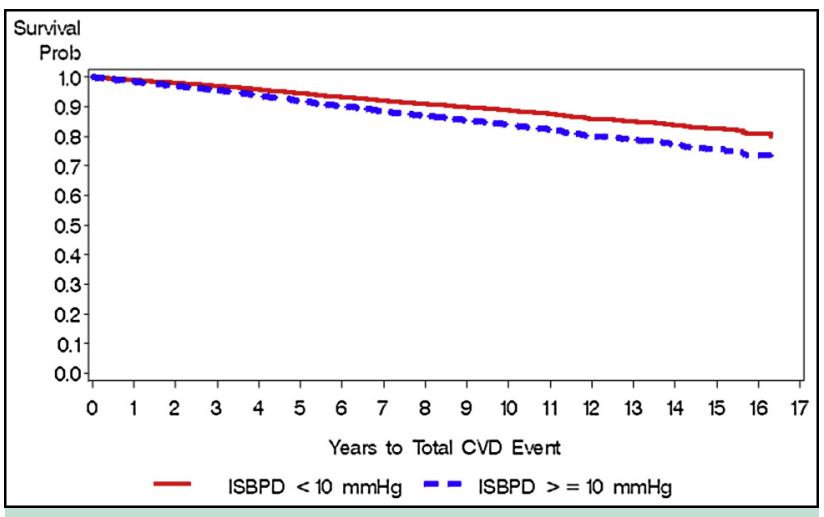

Figure 2 Age- and sex-adjusted Kaplan-Meier plot for incident cardiovascular events in 3390 participants with or without an interarm systolic blood pressure difference (ISBPD) $\geq 10 \mathrm{~mm} \mathrm{Hg}$ (log-rank test chi-squared $P<.0001$ ).

(Table 3). There was no significant sex interaction with ISBPD. Adjustment for familial clustering did not materially alter the results.

We examined model performance and the utility of ISBPD in reclassification of overall risk for incident cardiovascular disease. Both the c-statistic for a multivariable model that included ISBPD and a multivariable model that did not include ISBPD were 0.74 (95\% CI, 0.72-0.76). Category-based net reclassification for improvement changed significantly with ISBPD (NRI $0.021 ; P=.02$ ). The net reclassification is presented in Table 4.

\section{DISCUSSION}

In this large, prospective, community-based cohort of middle-age men and women free of cardiovascular disease, an increased ISBPD was found to be present in nearly $10 \%$ of individuals, and is associated with increased levels of traditional cardiovascular risk factors. Furthermore, an increased ISBPD is associated with an increased risk for incident cardiovascular events, independent of traditional cardiovascular risk factors. This association is consistent when ISBPD was examined as a binary and as a continuous variable, and showed a positive linear trend across categories of increasing ISBPD. This is the first communitybased cohort study to show such an association.

While we did not examine the anatomical correlates of increased ISBPD, our findings are consistent with previous publications linking subclavian artery stenosis to increased cardiovascular risk ${ }^{3,28}$ and are indirectly consistent with studies linking increased ISBPD with subclavian artery stenosis. Indeed, ISBPD $>10 \mathrm{~mm} \mathrm{Hg}$ has been shown to have a high sensitivity, ${ }^{29,30}$ yet a low specificity ${ }^{31}$ for angiographically significant subclavian artery stenosis. Furthermore, in 2 population-based studies, subclavian artery stenosis has been found to correlate with conventional risk factors for atherosclerosis. ${ }^{6,16}$ While a recent metaanalysis did not find a correlation between an ISBPD $\geq 15$ 
Table 2 Hazard Ratios of an Incident Cardiovascular Event or Mortality According to ISBPD Category

\begin{tabular}{|c|c|c|c|}
\hline & BP Category & $\begin{array}{l}\text { Incident CVD } \\
\text { HR }(95 \% \text { CI })\end{array}$ & $\begin{array}{l}\text { Mortality } \\
\text { HR }(95 \% \text { CI })\end{array}$ \\
\hline \multicolumn{4}{|c|}{ Primary analysis for ISBPD $\geq 10 \mathrm{~mm} \mathrm{Hg:}$} \\
\hline & $\geq 10 \mathrm{~mm} \mathrm{Hg}$ & $1.38(1.09-1.75)$ & $1.02(0.76-1.38)$ \\
\hline \multirow[t]{2}{*}{ Framingham 10-year risk score } & $<10$ referent & 1.00 & 1.00 \\
\hline & $\geq 10 \mathrm{~mm} \mathrm{Hg}$ & $1.38(1.09-1.75)$ & $0.98(0.72-1.33)$ \\
\hline \multirow[t]{4}{*}{ MV adjusted* } & $<10$ referent & 1.00 & 1.00 \\
\hline & 10 to $<15 \mathrm{~mm} \mathrm{Hg}$ & $1.36(1.04-1.77)$ & $0.99(0.71-1.39)$ \\
\hline & $\geq 15 \mathrm{~mm} \mathrm{Hg}$ & $1.47(0.93-2.32)$ & $1.11(0.62-1.99)$ \\
\hline & Trend $P$-value & .009 & .82 \\
\hline Framingham 10 -year risk score & $<10$ referent & 1.00 & 1.00 \\
\hline
\end{tabular}

$\mathrm{BP}=$ blood pressure; $\mathrm{CI}=$ confidence interval; $\mathrm{CVD}=$ cardiovascular disease; HR = hazard ratio; ISBPD = interarm systolic blood pressure difference; MV = multivariable.

*Adjusted for age, sex, smoking status, diabetes mellitus, hypertension, high-density lipoprotein cholesterol/total cholesterol ratio, and cholesterol treatment.

$\mathrm{mm} \mathrm{Hg}$ and a known pre-existing diagnosis of cardiovascular disease when pooling 6 studies, ${ }^{20}$ subclavian artery stenosis has been shown to correlate with ischemic heart disease as detected by stress myocardial single-photon emission computed tomography. ${ }^{32}$

In 6743 participants free of clinical cardiovascular disease in the Multi-Ethnic Study of Atherosclerosis (MESA) an ISBPD $\geq 15 \mathrm{~mm} \mathrm{Hg}$ was found in $307(4.5 \%)$ and correlated with subclinical measures of cardiovascular disease. ${ }^{3}$ Consistent with our findings, the MESA participants with an increased ISBPD tended to be older, more likely to have diabetes mellitus and hypertension, and were more obese than participants with a normal ISBPD. An increased ISBPD was found to correlate with markers of atherosclerosis including peripheral artery disease (ankle-brachial index $\leq 0.9$ ), an increase in carotid intima-media thickness, and a high coronary calcium score. Mortality and cardiovascular events were not assessed.

Several small prospective studies in cohorts of selected participants and a single meta-analysis previously described

Table 3 Standardized ISBPD: Risk-factor-adjusted Relative Risk of a First Cardiovascular Event According to ISBPD

\begin{tabular}{|c|c|c|}
\hline Stratification & $\operatorname{HR}(95 \% \mathrm{CI})^{*}$ & $P$-Value \\
\hline MV adjusted $\dagger$ & $1.07(1.00-1.14)$ & .04 \\
\hline Framingham 10 year risk score & $1.08(1.02-1.15)$ & .02 \\
\hline \multicolumn{3}{|c|}{$\begin{array}{l}\text { CI }=\text { confidence interval; HR }=\text { hazard ratio; ISBPD = interarm } \\
\text { systolic blood pressure difference; MV = multivariable. } \\
\text { *For every 1-SD-unit increase in blood pressure. } \\
\quad \text { †Adjusted for age, sex, smoking status, diabetes mellitus, hyper- } \\
\text { tension, high-density lipoprotein cholesterol/total cholesterol ratio and } \\
\text { cholesterol treatment. }\end{array}$} \\
\hline
\end{tabular}

an association between ISBPD and both all-cause and cardiovascular mortality, ${ }^{14,17,18,20}$ however, an association with cardiovascular disease in those studies was inconsistent. ${ }^{20}$ In a prospective study of 230 hypertensive patients (mean age 68.1 years) who were followed for a median of 9.8 years in a primary care setting, $55(23 \%)$ had an ISBPD $\geq 10 \mathrm{~mm}$ Hg. ${ }^{14}$ An ISBPD $\geq 10 \mathrm{~mm} \mathrm{Hg}$ was associated with incident CV events and with all-cause mortality, and there also was an increase of 5\%-6\% in the incidence of mortality for each 1-mm-Hg increase in ISBPD. In another prospective cohort recruited from 2 clinics, 421 consecutive patients (mean age 62.9 years), $95 \%$ of whom were men, were followed for a median of 5.6 years. Mortality was $31 \%$ and associated with an increase in ISBPD. ${ }^{18}$ In contrast, overall mortality in our study occurred in $12.2 \%$ over a longer period of time. Incident cardiovascular disease was not reported in this study. These 2 studies were small and selective, focusing on patients with hypertension or renal disease, making them less useful for drawing general conclusions. Adequately, in the secondary analysis in the subset of our study participants with hypertension, we observed a significant association between ISBPD and incident cardiovascular events. Finally, in another study combining 3 cohorts, 1778 subjects were recruited from both noninvasive vascular diagnostic laboratories and the community, and followed for a median of 9.4 years. ${ }^{17}$ In a fully adjusted model accounting for cardiovascular risk factors and for baseline cardiovascular disease, an ISBPD $\geq 15 \mathrm{~mm} \mathrm{Hg}$ demonstrated only increasing but not significant hazards for cardiovascular mortality (HR 1.43; 95\% CI, 0.93-2.18). Incident cardiovascular disease was again not reported, the study population was enriched with high-risk patients being evaluated in vascular laboratories, and cause of death was adjudicated based on death certificate data. As expected, our study 
Table 4 Net Reclassification Stratified by CVD Events

No CVD Event $(n=2783)$

Incident CVD Event $(\mathrm{n}=593)$

Risk Category with ISBPD

\begin{tabular}{lccccrr}
\hline Risk Category without ISBPD & $<6 \%$ & $6 \%-20 \%$ & $>20 \%$ & $<6 \%$ & $6 \%-20 \%$ & $>20 \%$ \\
\hline$<6 \%$ & 543 & 16 & 0 & 177 & 6 & 0 \\
$6 \%-20 \%$ & 23 & 1465 & 20 & 5 & 260 & 7 \\
$>20 \%$ & 0 & 26 & 690 & 0 & 4 & 134 \\
\hline
\end{tabular}

CVD = cardiovascular disease; ISBPD = interarm systolic blood pressure difference.

participants were of lower risk, and it is not surprising that our HR for cardiovascular events were lower. Our lowerrisk population also may have been underpowered to detect a mortality association with ISBPD. Post hoc power calculations revealed that with 489/3390 deaths, 50 of which occurred in the ISBPD $\geq 10 \mathrm{~mm} \mathrm{Hg}$, we only had $22 \%$ and $76 \%$ power to detect HRs of 1.2 and 1.5, respectively. For the association with mortality, our HR was low and not significant. Furthermore, of the deaths in our cohort, most were not cardiovascular disease related, further limiting our ability to assess an association with ISBPD.

Potential limitations of the present study include sequential, as opposed to simultaneous, blood pressure measurements, ${ }^{2,7,33-35}$ and blood pressure measurements during one encounter as opposed to an average of separate encounters. ${ }^{34,36,37}$ Nonetheless, in a cohort in which the prevalence of ISBPD $>10 \mathrm{~mm} \mathrm{Hg}$ was $40 \%$, the ISBPD was roughly stable, despite repeated measurements, ${ }^{18}$ and in 2 studies comparing sequential and simultaneous measurements, the ISBPD differences were well below the $10 \mathrm{~mm} \mathrm{Hg}$ threshold that we have used. ${ }^{35,38}$ Given that our results have shown significant and consistent prognostic value, they are unlikely to represent random variation. Furthermore, sequential blood pressure measurement is the accepted method of measuring arm blood pressures in clinical practice, ${ }^{39}$ making our results clinically applicable. Strengths of the present study include its large community-based sample, a comprehensive directly measured covariate assessment, and validated cardiovascular events and deaths. Finally, the significant category-based net reclassification improvement index suggests statistically significant, albeit small, enhancement of incident cardiovascular event prediction beyond traditional risk factors by considering ISBPD.

In conclusion, in this community-based cohort, an ISBPD was common and was associated with a significant increase in risk for cardiovascular events even after adjustment for cardiovascular risk factors. Even modest differences in clinically measured systolic blood pressures in the upper extremities reflect on cardiovascular risk. This study supports the potential value of identifying the ISBPD as a simple clinical indicator of increased cardiovascular risk. Blood pressure is easily obtained in an office setting, and our findings support recommendations for measurement of blood pressure in both arms, both for accurate blood pressure detection and for detection of ISBPD.
Our findings support research to expand clinical use of this simple measurement.

\section{ACKNOWLEDGEMENTS}

The Framingham Heart Study is supported by the NHLBI Framingham Heart Study (contract N01-HC-25195). Analysis for this project was supported by the NHLBI Division of Intramural Research.

\section{References}

1. National Institute for Health and Clinical Excellence. Hypertension: Clinical Management of Primary Hypertension in Adults. London: National Institute for Health and Clinical Excellence; 2011.

2. Orme S, Ralph SG, Birchall A, Lawson-Matthew P, McLean K, Channer KS. The normal range for inter-arm differences in blood pressure. Age Ageing. 1999;28:537-542.

3. Aboyans V, Kamineni A, Allison MA, et al. The epidemiology of subclavian stenosis and its association with markers of subclinical atherosclerosis: the Multi-Ethnic Study of Atherosclerosis (MESA). Atherosclerosis. 2010;211:266-270.

4. Shock NW, Ogden E. The differences between blood pressure measurements obtained simultaneously in both arms. Exp Physiol. 1940;30: 155-162.

5. Lane D, Beevers M, Barnes N, et al. Inter-arm differences in blood pressure: when are they clinically significant? J Hypertens. 2002;20: 1089-1095.

6. Shadman R, Criqui MH, Bundens WP, et al. Subclavian artery stenosis: prevalence, risk factors, and association with cardiovascular diseases. J Am Coll Cardiol. 2004;44:618-623.

7. Clark CE, Powell RJ. The differential blood pressure sign in general practice: prevalence and prognostic value. Fam Pract. 2002;19: 439-441.

8. Mendelson G, Nassimiha D, Aronow WS. Simultaneous measurements of blood pressures in right and left brachial arteries. Cardiol Rev. 2004;12:276-278.

9. Clark CE, Greaves CJ, Evans PH, Dickens A, Campbell JL. Inter-arm blood pressure difference in type 2 diabetes: a barrier to effective management? Br J Gen Pract. 2009;59:428-432.

10. National Heart, Lung, and Blood Institute. The Seventh Report of the Joint National Committee on Prevention, Detection, Evaluation and Treatment of High Blood Pressure - Complete Report. Available at: http://www. nhlbi.nih.gov/guidelines/hypertension/jnc7full.htm. Accessed March 22, 2013.

11. Rueger MJ. Blood pressure variations in the two arms. Ann Intern Med. 1951;35:1023-1027.

12. Mancia G, De Backer G, Dominiczak A, et al; the task force for the management of arterial hypertension of the European Society of Hypertension, the task force for the management of arterial hypertension of the European Society of Cardiology. 2007 Guidelines for the management of arterial hypertension: the Task Force for the Management of Arterial Hypertension of the European Society of Hypertension 
(ESH) and of the European Society of Cardiology (ESC). Eur Heart J. 2007;28:1462-1536.

13. Giles TD, Egan P. Inter-arm difference in blood pressure may have serious research and clinical implications. J Clin Hypertens (Greenwich). 2012;14:491-492.

14. Clark CE, Taylor RS, Shore AC, Campbell JL. The difference in blood pressure readings between arms and survival: primary care cohort study. BMJ. 2012;344:e1327.

15. Clark CE, Campbell JL, Powell RJ, Thompson JF. The inter-arm blood pressure difference and peripheral vascular disease: cross-sectional study. Fam Pract. 2007;24:420-426.

16. Kimura A, Hashimoto J, Watabe D, et al. Patient characteristics and factors associated with inter-arm difference of blood pressure measurements in a general population in Ohasama, Japan. J Hypertens. 2004:22:2277-2283.

17. Aboyans V, Criqui MH, McDermott MM, et al. The vital prognosis of subclavian stenosis. J Am Coll Cardiol. 2007;49:1540-1545.

18. Agarwal R, Bunaye Z, Bekele DM. Prognostic significance of betweenarm blood pressure differences. Hypertension. 2008;51:657-662.

19. Pesola GR, Pesola HR, Lin M, Nelson MJ, Westfal RE. The normal difference in bilateral indirect blood pressure recordings in hypertensive individuals. Acad Emerg Med. 2002;9:342-345.

20. Clark CE, Taylor RS, Shore AC, Ukoumunne OC, Campbell JL. Association of a difference in systolic blood pressure between arms with vascular disease and mortality: a systematic review and meta-analysis. Lancet. 2012;379:905-914.

21. Kannel WB, Feinleib M, McNamara PM, Garrison RJ, Castelli WP. An investigation of coronary heart disease in families. The Framingham offspring study. Am J Epidemiol. 1979;110:281-290.

22. Splansky GL, Corey D, Yang Q, et al. The third generation cohort of the National Heart, Lung, and Blood Institute's Framingham Heart Study: design, recruitment, and initial examination. Am J Epidemiol. 2007; 165:1328-1335.

23. Hubert HB, Feinleib M, McNamara PM, Castelli WP. Obesity as an independent risk factor for cardiovascular disease: a 26-year follow-up of participants in the Framingham Heart Study. Circulation. 1983;67: 968-977.

24. Murabito JM, Evans JC, Nieto K, Larson MG, Levy D, Wilson PW. Prevalence and clinical correlates of peripheral arterial disease in the Framingham Offspring Study. Am Heart J. 2002;143:961-965.

25. D’Agostino RB Sr, Vasan RS, Pencina MJ, et al. General cardiovascular risk profile for use in primary care: the Framingham Heart Study. Circulation. 2008;117:743-753.

26. Wilson PW, D'Agostino RB, Levy D, Belanger AM, Silbershatz H, Kannel WB. Prediction of coronary heart disease using risk factor categories. Circulation. 1998;97:1837-1847.
27. Pencina MJ, D'Agostino RBS, Steyerberg EW. Extensions of net reclassification improvement calculations to measure usefulness of new biomarkers. Stat Med. 2011;30:11-21.

28. Baribeau Y, Westbrook BM, Charlesworth DC, Hearne MJ, Bradley WA, Maloney CT. Brachial gradient in cardiac surgical patients. Circulation. 2002;106:I11-I13.

29. Korns HM, Guinand PH. Inequality of blood pressure in the brachial arteries, with especial reference to disease of the arch of the aorta. J Clin Invest. 1933;12:143-154.

30. Osborn LA, Vernon SM, Reynolds B, Timm TC, Allen K. Screening for subclavian artery stenosis in patients who are candidates for coronary bypass surgery. Catheter Cardiovasc Interv. 2002;56:162-165.

31. English JA, Carell ES, Guidera SA, Tripp HF. Angiographic prevalence and clinical predictors of left subclavian stenosis in patients undergoing diagnostic cardiac catheterization. Catheter Cardiovasc Interv. 2001;54:8-11.

32. Igarashi $\mathrm{Y}$, Chikamori $\mathrm{T}$, Tomiyama $\mathrm{H}$, et al. Clinical significance of interarm pressure difference and ankle-brachial pressure index in patients with suspected coronary artery disease. J Cardiol. 2007;50:281-289.

33. Singer AJ, Hollander JE. Blood pressure. Assessment of interarm differences. Arch Intern Med. 1996;156:2005-2008.

34. Verberk WJ, Kessels AG, Thien T. Blood pressure measurement method and inter-arm differences: a meta-analysis. Am J Hypertens. 2011;24:1201-1208.

35. Cassidy $\mathrm{P}$, Jones $\mathrm{K}$. A study of inter-arm blood pressure differences in primary care. J Hum Hypertens. 2001;15:519-522.

36. Hashimoto F, Hunt WC, Hardy L. Differences between right and left arm blood pressures in the elderly. West J Med. 1984;141:189-192.

37. Eguchi K, Yacoub M, Jhalani J, Gerin W, Schwartz JE, Pickering TG. Consistency of blood pressure differences between the left and right arms. Arch Intern Med. 2007;167:388-393.

38. Harrison EG Jr, Roth GM, Hines EA Jr. Bilateral indirect and direct arterial pressures. Circulation. 1960;22:419-436.

39. Clark CE. Inter-arm blood pressure measurement needs to be practical and accurate. Am J Hypertens. 2011;24:1189-1190.

Funding: The Framingham Heart Study is supported by the National Heart, Lung and Blood Institute (NHLBI) Framingham Heart Study (contract N01-HC-25195). Analysis for this project was supported by the NHLBI Division of Intramural Research.

Authorship: All authors had access to the data; other author roles were as follows: IW, study design, literature search, data interpretation, writing; PG, study design, data analysis, writing; CJD, study design, data interpretation, editing; MRJ, study design, data interpretation, editing; JMM, study design, data interpretation, editing. 